\title{
BMJ Open Short-term weight gain among preschool children in rural Burkina Faso: a secondary analysis of a randomised controlled trial
}

\author{
Elena G Dennis, ${ }^{1}$ Ali Sie,${ }^{2}$ Lucienne Ouermi, ${ }^{2}$ Clarisse Dah, ${ }^{2}$ \\ Charlemagne Tapsoba, ${ }^{2}$ Pascal Zabre, ${ }^{2}$ Till Bärnighausen, ${ }^{3,4,5}$ Kieran S O'Brien, ${ }^{1}$ \\ Elodie Lebas, ${ }^{1}$ Jeremy D Keenan, ${ }^{\oplus 1,6}$ Catherine E Oldenburg ${ }^{\oplus 1,6,7}$
}

To cite: Dennis EG, Sie A, Ouermi L, et al. Shortterm weight gain among preschool children in rural Burkina Faso: a secondary analysis of a randomised controlled trial. BMJ Open 2019;9:e029634. doi:10.1136/ bmjopen-2019-029634

- Prepublication history for this paper is available online. To view these files, please visit the journal online (http://dx.doi org/10.1136/bmjopen-2019029634).

Received 02 February 2019 Revised 28 June 2019 Accepted 11 July 2019
Check for updates

(C) Author(s) (or their employer(s)) 2019. Re-use permitted under CC BY-NC. No commercial re-use. See rights and permissions. Published by BMJ.

For numbered affiliations see end of article.

Correspondence to Dr Catherine E Oldenburg; catherine.oldenburg@ucsf.edu

\section{ABSTRACT}

Objectives Nutrition has profound effects on children's health outcomes and is linked to weight gain and cognitive development. We used data from a randomised controlled trial to evaluate the prospective associations between dietary, socioeconomic and demographic factors and short-term weight gain during the lean season in a rural area of Burkina Faso.

Design Prospective cohort data arising from a randomised controlled trial of the effect of antibiotic distribution on child growth and intestinal microbial diversity.

Setting Two rural communities in Nouna District, Burkina Faso.

Participants 246 children aged 6-59 months living in the study communities were enrolled in the study.

Primary and secondary outcome

measures Anthropometric measurements, including weight and height, were obtained at baseline and 1 month. Results of 246 children, the median weight for wasted children at baseline (weight-for-height z-score $<-2$ ) was $9.7 \mathrm{~kg}$ (IQR 8.65-10.8) and the weight of non-wasted children was $12.8 \mathrm{~kg}$ (IQR 10.9-14.75). Food insecurity was significantly associated with decreased weight gain velocity (mean difference $-0.03 \mathrm{~g} / \mathrm{kg} / \mathrm{day}, 95 \% \mathrm{Cl}-0.06$ to $-0.006, p=0.04$ ).

Conclusion Experiences of household food insecurity before the beginning of the lean season were associated with decreased weight gain in children in rural Burkina Faso during the lean season, although the mean difference was small. Understanding the relationship between timing of food insecurity and anthropometric outcomes may help to develop policies and health programme that address both of these issues.

Trial registration number NCT03187834.

\section{BACKGROUND}

Undernutrition is implicated in $50 \%$ of child deaths every year. ${ }^{1}$ Nutrition has profound effects on health throughout the human life course and is inextricably linked to weight gain and cognitive development during early childhood. ${ }^{2}$ In rural settings with insufficient resources, children are at greater risk of failing
Strengths and limitations of this study

- We used prospective data collected during the lean season in rural Burkina Faso to evaluate factors associated with weight gain in preschool children.

- Data were collected during the lean season in Burkina Faso, when children are at particularly high risk of malnutrition.

- Data were collected in a standardised fashion by trained anthropometrists.

- Limitations include the relatively small sample size and low prevalence of wasting, which may limit power particularly for analyses of factors associated with wasting.

to reach their full growth and development potential. $^{2}$ Several cross-sectional studies have evaluated the underlying factors that contribute to malnutrition in an attempt to improve strategies to address the prevalence of child undernutrition, focusing primarily on nutrition-related determinants of growth. These studies identified several potential modifiable risk factors for undernutrition.

Dietary diversity is critical to ensure sufficient micronutrient intake. ${ }^{3}$ Numerous studies have linked dietary diversity to nutritional status in children, ${ }^{4}$ finding that greater diversity is associated with a greater likelihood of meeting nutrient requirements and positive health outcomes. ${ }^{3}$ In a study using data from 11 Health and Demographic surveys, dietary diversity was significantly associated with increased height-for-age z-score in seven countries, ${ }^{3}$ indicating that dietary diversity is important for a child's long-term nutritional status.

Food insecurity is associated with lower dietary diversity and poorer child health outcomes. ${ }^{5}$ Food insecurity has a wide range of causes, including low socioeconomic status 
and seasonal variation in food availability. ${ }^{6}$ In sub-Saharan Africa and particularly in rural, agrarian areas, the dependence on rainfall and the abundance of subsistence farming create seasonal variations in food availability. ${ }^{7}$ In the Sahel region, many experience a 'lean season' during seasonal rains, typically April to August. Conversely, these populations also experience a drier post-harvest season from January to March. ${ }^{7}$ Seasonal variation in rainfall contributes to an increase in morbidity such as malaria, diarrhoea and upper and lower respiratory infections. These diseases can impact a child's nutritional status by increasing their nutritional needs and decreasing their appetite. ${ }^{7}$ A study conducted in Burkina Faso found that the diversity of household diets was greater throughout all seasons with higher food expenditures, greater crop production and sale and with a household head educated at the post-secondary level. ${ }^{8}$

Although multiple cross-sectional studies have evaluated the association between dietary diversity and sociodemographic factors and nutritional status, fewer studies have examined factors influencing weight gain in young children prospectively. Cross-sectional studies are limited by inability to determine temporality, and potential predictors may be influenced by outcomes of interest. Here, we used data from a randomised controlled trial to evaluate the prospective associations between dietary, socioeconomic and demographic factors to identify possible modifiable risk factors for short-term weight gain during the beginning of the lean season in a rural area of Burkina Faso.

\section{METHODS}

\section{Study setting}

This study was conducted in the Nouna Health and Demographic Surveillance Site (HDSS) in the sub-Sahelian villages of Kamadena and Dara in rural northwestern Burkina Faso. The HDSS represents roughly one-quarter of the Nouna Health District in terms of surface and population, and the population is primarily made up of cattle keeps and subsistence farmers. ${ }^{9}$ This study was conducted from July to August 2017, during the beginning of the rainy season in Burkina Faso which lasts from July to October. The rainy season coincides with peak malaria and malnutrition in the Sahel and sub-Sahel.

Data for the present analysis arose from a randomised controlled trial designed to assess the effect of commonly used childhood antibiotics on the composition of the intestinal microbiome and anthropometry. ${ }^{10}{ }^{11}$ In the parent trial, children ages 6-59 months in households with two to three children at the most recent HDSS census were eligible for participation. Households were excluded if one of the children was unable to participate in the baseline assessment, due to illness or absence. If the household had two or three children, they were all enrolled and anthropometric measures were taken. Children's caregivers completed assessments at the beginning of the study. After the baseline assessment, children were randomised in a 1:1:1:1 fashion to a 5 day course of placebo, amoxicillin, azithromycin or cotrimoxazole. ${ }^{10}$ All treatments were directly observed by study staff and administered as paediatric oral suspension. Children were followed for 35 days from enrolment for anthropometric outcomes. ${ }^{11}$ All data were collected and managed in CommCare (Dimagi, Cambridge, Massachusetts, USA).

\section{Anthropometric assessment}

Height, weight and mid-upper-arm circumference (MUAC) measurements were assessed at baseline and at 35 days after enrolment. Children were weighed standing if able or in the arms of a caregiver, with heavy garments and jewellery removed. Recumbent length was measured in children $<24$ months of age and standing height in children $>24$ months of age (Seca 874 flat floor scale). Height and weight measurements were taken three times and the median for each measure was used for analysis. The median of the three measurements was used to avoid undue influence of outlying or implausible values. MUAC was measured a single time. Weight-for-height z-score (WHZ) and weight-for-age z-score (WAZ) were calculated based on 2006 WHO Child Growth Standards. ${ }^{12}$ Change in weight, defined as the mean difference, and weight gain velocity, defined as grams per kilogram per day were also calculated. Wasting and underweight were defined as $\mathrm{WHZ}$ and $\mathrm{WAZ}<-2 \mathrm{SD}$, respectively.

\section{Predictors}

Age and sex were extracted from the HDSS database. Dietary diversity, food insecurity status, breastfeeding status, healthcare facility visits and animal and latrine ownership were assessed at baseline by asking caregivers in their local dialect a variety of questions for each topic. Breastfeeding status was determined by asking the caregiver if the child was currently breast feeding, and if so if the child was exclusively breast feeding. Dietary diversity was evaluated using a questionnaire that asked if the child had eaten a series of 11 food groups in the past 7 days, including grains (millet, rice, sorghum), vitamin-A abundant foods (carrots, sweet potatoes, squash), greens, mangoes/papayas, other fruits, vegetables, proteins (meat, poultry or fish), eggs, legumes, dairy products (milk, yoghourt, cheese, etc), fats (coconut milk, butter, oil, etc), sugary beverages, fortified foods and ready-to-eat supplementary or therapeutic foods. ${ }^{413}$ The answers were made into a composite dietary diversity score by categorising the food groups into seven unique food groups, including starch, vitamin A-rich foods, other fruits and vegetables, animal protein (eg, meat, eggs, poultry, fish), legumes, dairy and fat (eg, oil, butter, other fat). ${ }^{3}$ We then summed the number of food groups reported for each child by the caregiver. The possible range was 0 , for children who ate none of the food groups, to 11, for children who ate foods from every food group. For each household, caregivers reported on three questions regarding food insecurity, including the number of times in the past 4 weeks the caregiver worried about not having 
enough food in the household, if a member had gone to bed hungry in the past 4 weeks and if a member had to eat limited amount of food because lack of resource in the previous 4 weeks. ${ }^{4}{ }^{14}$ Breastfeeding status was measured by asking caregivers if the child was breast fed and if so, if the child was exclusively breast fed. Caregivers reported on the number of poultry, goats/sheep and cows that their household owned. The total number of animals was summed. Finally, each caregiver reported whether they had visited a health facility for their child in the past 30 days and on the sanitation installation most commonly used by their household, categorised as none (open defection), latrine with slab or latrine without slab. Finally, the child's randomisation arm was included as a covariate in all models.

\section{Sample size}

The sample size calculation was based on the primary outcome of the trial, Simpson's $\alpha$ diversity. A sample size of 30 children per arm was estimated to provide at least $80 \%$ power to detect a 1.5-unit difference in Simpson's $\alpha$ diversity based on a previous study in Niger. ${ }^{15}$

\section{Statistical methods}

Descriptive statistics were calculated with medians and IQRs for continuous variables and proportions for categorical variables. To assess predictors of weight gain in the 1-month period, a bivariate model was built for each anthropometric outcome (WHZ, WAZ, change in weight in grams, wasting and underweight status at day 35, and $\mathrm{g} / \mathrm{kg} /$ day) and each baseline predictor (including age, sex, dietary diversity score, food insecurity score, latrine ownership, animal ownership, healthcare facility use and breastfeeding status). One model was built per outcome. Linear regression analyses were performed for the continuous outcomes and a logistic regression analysis was run for the dichotomous outcome. Multivariable models were then built for each anthropometric outcome with all candidate predictor variables, including child's sex, age, baseline WHZ, food insecurity, healthcare facility usage, dietary diversity score, breastfeeding status, animal ownership and latrine ownership. SEs of all regression models were adjusted for clustering at the household level. Children with implausible weight changes between baseline and 1-month measurements (gained or lost more than $2 \mathrm{~kg}$ ) were assumed to be data entry errors (eg, the wrong child was measured), and were excluded from analyses. All analyses were performed in Stata V.15.1 (StataCorp).

\section{Patient and public involvement}

This study recruited a population-based sample of the general population, and thus no patients were involved in the study. Leaders of the study communities were involved in informing residents about the study, recruiting children and families to participate, and facilitating follow-up visits.

\section{RESULTS}

For the trial, 165 households were assessed for eligibility and 41 were excluded because two children were not present in the household. The remaining 124 households were eligible for inclusion and were enrolled in the study. ${ }^{11}$ A total of 248 children were enrolled in the study, of whom 233 had eligible anthropometric measurements at baseline and 4 weeks after treatment. Table 1 lists baseline descriptive statistics from the analysis. From the total number of children, $49.6 \%$ were female and the median age was 37 months (IQR 23-49). The mean baseline weight for children with WHZ $<-2$ was $9.7 \mathrm{~kg}$ (SD 1.3) compared with non-wasted $12.8 \mathrm{~kg}$ (SD 2.8) in non-wasted children. Approximately $50 \%$ of caregivers with wasted children reported that they visited a healthcare facility in the past 30 days. The median dietary diversity score was six for both groups, non-wasted (IQR 4-7) and wasted (IQR 5-7). Households with a wasted child owned a median of 24.5 animals (IQR 6-54) while the families of non-wasted children owned a median of 13 (6 to 28 ). More wasted children were breast fed (35.7\%) compared with non-wasted children $(21.1 \%)$.

From baseline to 1 month, 219 non-wasted children gained a mean of $334 \mathrm{~g}$ (SD 485), and weight gain velocity was $0.82 \mathrm{~g} / \mathrm{kg} /$ day (SD 1.2). The median WHZ at 1 month after baseline was -0.37 SD (SD 0.98 ), and $6.0 \%$ of children were wasted. Caregivers of five children reported that their child received antibiotics outside of the study treatment during the course of the study.

Table 2 lists a series of bivariate and multivariable models depicting the association between candidate predictor variables and WHZ and wasting status 1 month after baseline. The only significant predictor of WHZ at 1 month was baseline WHZ. In a bivariate model, children who had visited the health facility in the past month had increased odds of wasting (adjusted OR 5.66, 95\% CI 1.85 to $17.3, \mathrm{p}=0.001$ ), and children living in households owning greater numbers of animals had increased odds of wasting (adjusted OR 1.01 per one additional animal owned by the household, 95\% CI 1.00 to $1.02, \mathrm{p}=0.005$ ). However, wasting at 1 month was relatively uncommon and CIs were wide, and animal ownership was not significant in the multivariable models. There was a non-significant increase in risk of wasting in children living in households with higher levels of food insecurity (adjusted OR $1.32,95 \%$ CI 1.00 to $1.74, p=0.05$ ). No other variables were statistically significantly associated with WHZ or wasting 4 weeks after baseline.

Table 3 lists bivariate and multivariable models for the association between candidate predictor variables and weight change and weight gain velocity during the 1-month period. In the multivariable model, children in households with higher food insecurity scores had decreased weight gain velocity (mean difference -0.03 $\mathrm{g} / \mathrm{kg} /$ day per one-unit increase in food insecurity, $95 \%$ CI -0.06 to $-0.006, p=0.04$ ). Dietary diversity was not significantly associated with weight gain velocity (mean difference $-0.05 \mathrm{~g} / \mathrm{kg} /$ day for every one-unit increase 
Table 1 Baseline descriptive statistics of the study $(\mathrm{N}=246)$

\begin{tabular}{|c|c|c|c|}
\hline & $\begin{array}{l}\text { Not wasted (MUAC or } \\
\text { WHZ >-2) } \\
\mathrm{N}=232\end{array}$ & $\begin{array}{l}\text { Wasted (MUAC or WHZ } \\
<-2 \text { ) } \\
\mathrm{N}=14\end{array}$ & $\begin{array}{l}\text { Overall } \\
\mathrm{N}=246\end{array}$ \\
\hline Age, months, median (IQR) & $37.5(25-50)$ & $37(23-46)$ & $37(23-49)$ \\
\hline Female sex, n (\%) & $115(49.6)$ & $7(50.0)$ & $122(49.6)$ \\
\hline Male sex, $n(\%)$ & $117(50.4)$ & $7(50.0)$ & $124(50.4)$ \\
\hline Weight, kg, mean (SD) & $12.8(2.8)$ & $9.7(1.3)$ & $12.7(2.8)$ \\
\hline Height, cm, mean (SD) & $90.9(10.1)$ & $85.3(6.9)$ & $90.6(10.0)$ \\
\hline WHZ, mean (SD) & $-0.31(1.09)$ & $-2.3(0.50)$ & $-0.42(1.16)$ \\
\hline WAZ, mean (SD) & $-0.85(0.99)$ & $-2.41(0.80)$ & $-0.94(1.04)$ \\
\hline HAZ, mean (SD) & $-1.13(1.48)$ & $-1.56(1.16)$ & $-1.16(1.46)$ \\
\hline MUAC, mean (SD) & $15.2(1.10)$ & $13.7(0.72)$ & $15.2(1.14)$ \\
\hline $\begin{array}{l}\text { Number of times went to bed hungry due to not } \\
\text { enough food, last } 35 \text { days, median (IQR) }\end{array}$ & $0(0-0)$ & $0(0-3)$ & $0(0-0)$ \\
\hline Had limited food, n (\%) & $50(21.6)$ & $5(35.7)$ & $55(22.4)$ \\
\hline Went to bed hungry, last 35 days, $n$ (\%) & $28(12.1)$ & $2(14.9)$ & $30(12.2)$ \\
\hline Visited healthcare facility in past 30 days, $n(\%)$ & $32(14.2)$ & $7(50.0)$ & 39 (16.3) \\
\hline Dietary diversity score, median (IQR) & $6(4-7)$ & $6(5-7)$ & $6(4-7)$ \\
\hline Any breast feeding, $\mathrm{n}(\%)$ & $49(21.1)$ & $5(35.7)$ & $54(22.0)$ \\
\hline $\begin{array}{l}\text { Number of animals owned by household, } \\
\text { median (IQR) }\end{array}$ & $13(6-28)$ & $24.5(6-54)$ & $13(6-29.5)$ \\
\hline \multicolumn{4}{|l|}{ Household latrine ownership, $n(\%)$} \\
\hline Bush & $82(35.3)$ & $3(21.4)$ & $85(34.6)$ \\
\hline Slab & $70(30.2)$ & $7(50.0)$ & $77(31.3)$ \\
\hline No slab & $80(34.5)$ & $4(28.5)$ & $84(34.4)$ \\
\hline Change in weight, median (IQR) & $350(50-600)$ & $185(-50-500)$ & $310(50-600)$ \\
\hline Grams per kilogram per day, median (IQR) & $0.71(0.12-1.36)$ & $0.61(-0.14-1.44)$ & $0.70(0.12-1.37)$ \\
\hline Underweight at day $35, \mathrm{n}(\%)$ & $14(6.0)$ & $8(57.1)$ & $22(8.9)$ \\
\hline
\end{tabular}

HAZ, height-for-age z-score; MUAC, mid-upper-arm circumference; WAZ, weight-for-age z-score; WHZ, weight-for-height z-score;

in dietary diversity score, CI $95 \%$ ( -0.16 to $0.05, \mathrm{p}=0.29)$. A higher food insecurity score was also associated with reduced change in weight (mean difference $-12.2 \mathrm{~g}$ per 1-unit increase in food insecurity score, $95 \%$ CI -24.3 to $-0.03, \mathrm{p}=0.049$ ).

Table 4 lists bivariate and multivariable models for the association between candidate predictor variables and WAZ and underweight 4 weeks after baseline. Age was significantly associated with WAZ in the multivariable model (mean difference $-0.005 \mathrm{SD}$ per 1 month increase in age, $95 \%$ CI -0.009 to $-0.0008, p=0.02$ ). No other candidate predictors were statistically significantly associated with WAZ or underweight.

\section{DISCUSSION}

The purpose of the present study was to assess socioeconomic and dietary predictors of a child's short-term weight gain in a sub-Sahelian region of Burkina Faso to identify potential modifiable risk factors at the beginning of the lean season that may lead to better nutritional outcomes for preschool children. Food insecurity was the only independent predictor significantly associated with decreased weight gain velocity and change in weight. Food insecurity was measured over the 30 -day period prior to the baseline assessment which happened at the beginning of the lean season. These findings suggest that children in households experiencing food insecurity before the lean season are at higher risk of poor weight gain which could result in malnutrition and lead to serious consequences for their physical and cognitive development. ${ }^{16}$ These results are consistent with previous literature, which shows a negative association between higher food insecurity and lower dietary diversity with a child's nutritional status. ${ }^{39}$ These results could be explained by the lack of nutrient dense foods available during and before the lean season, as food insecurity before the lean season is likely predictive of food insecurity during the lean season. Previous studies indicate that during the lean season, staple dishes are more often bought ready-to-eat and usually contain fewer nutrients and raw ingredients in comparison to 




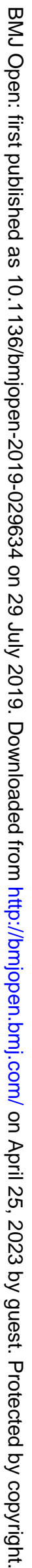




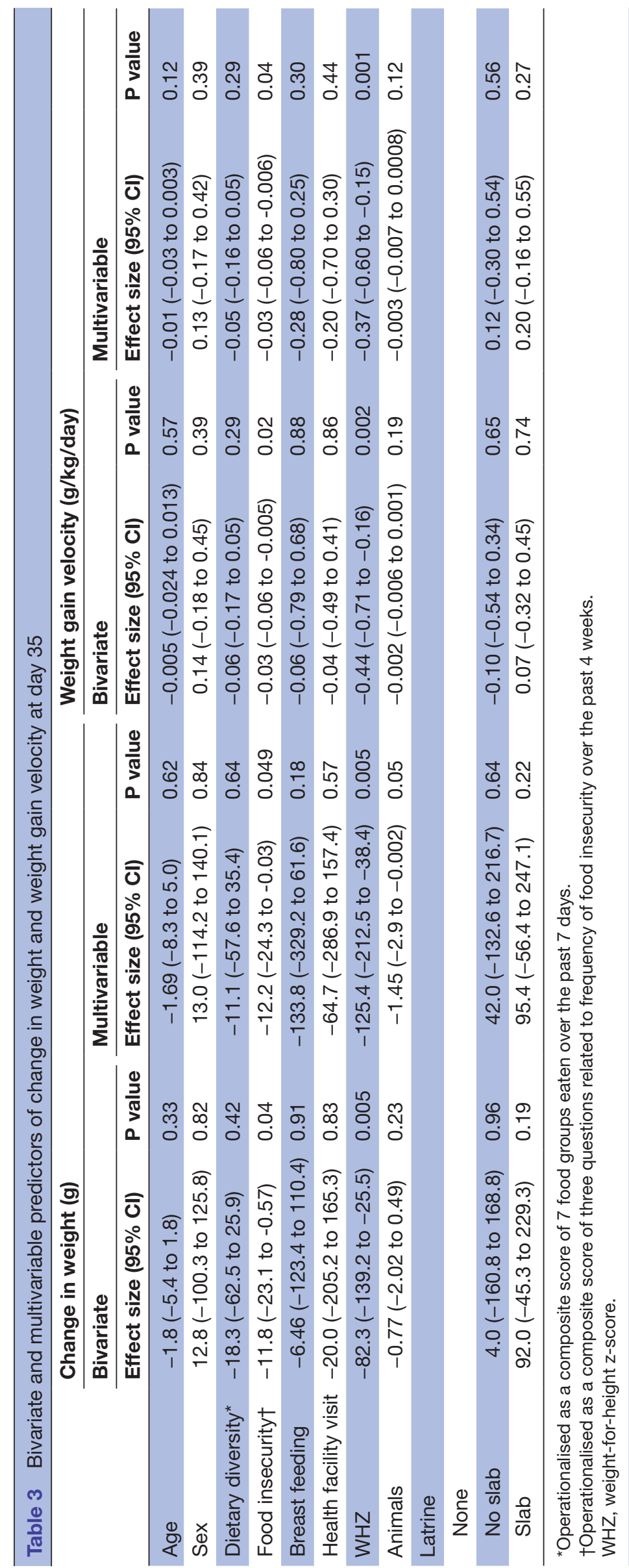

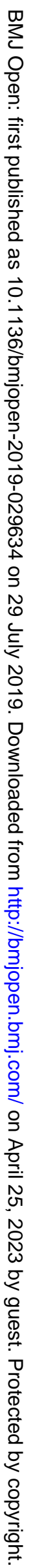




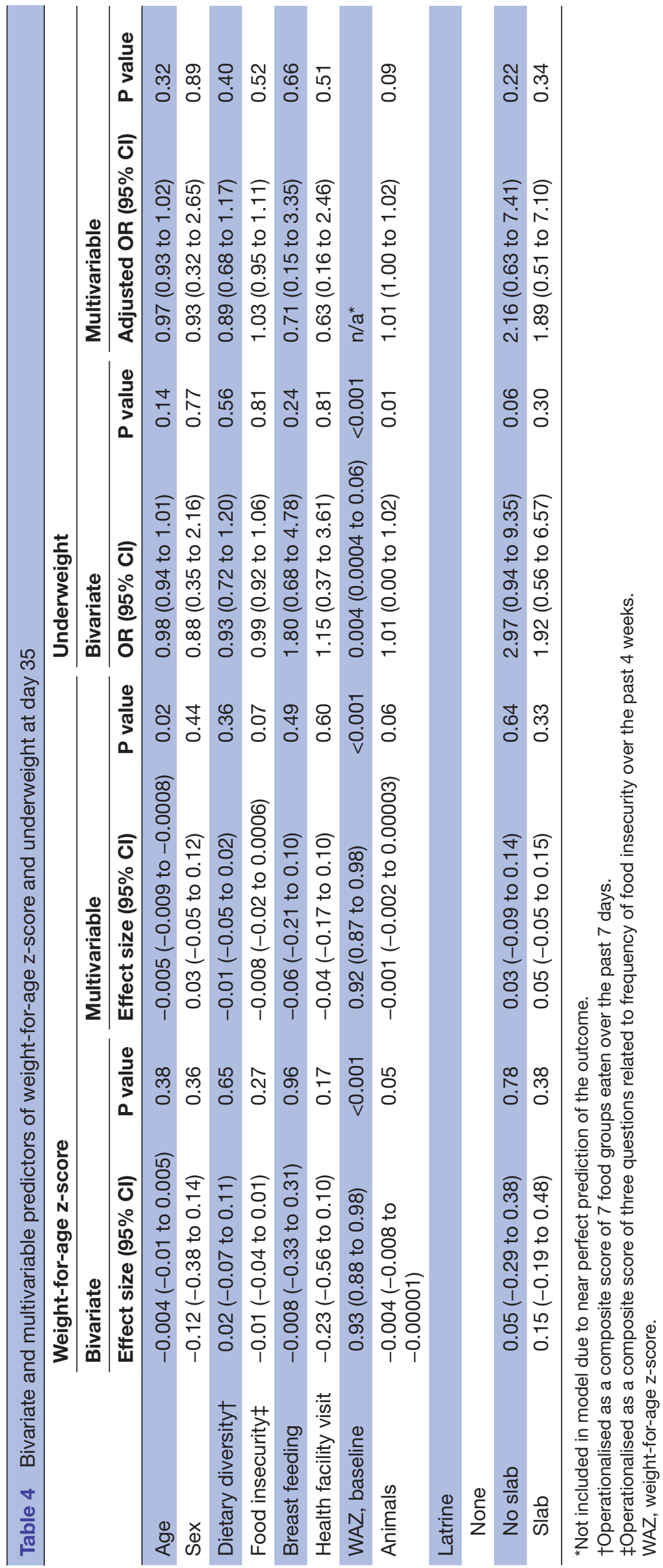

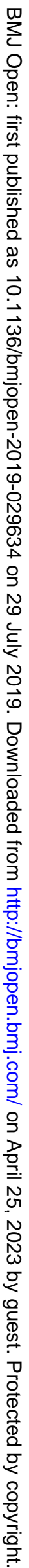


meals made during the Sahel's post-harvest season. ${ }^{17}$ The results of this study suggest that food insecurity, above and beyond other potential risk factors, is an important potentially modifiable risk factor for adverse nutritional outcomes. These findings underscore the importance of prioritising policies related to improving food security in areas with seasonal malnutrition, as experiences of food insecurity immediately before the beginning of the lean season may predispose children to worse outcomes during the course of the lean season. Interventions addressing food insecurity prior to the lean season, not only during the lean season, may help improve outcomes for children during this vulnerable time.

The prevalence of wasting and underweight was lower than expected at 1 month, ${ }^{18}$ limiting statistical power to detect risk factors for both conditions. Children who had visited a health facility had increased odds of wasting and reduced WHZ at day 35, although this was not statistically significant in multivariable models. This is likely reflective of parents seeking care for malnourished children, and reduced weight gain was likely related to sick children gaining less weight.

This study should be considered in the context of its limitations. First, the study collected data via caregiver report which could be subject to misclassification and bias. ${ }^{4}$ The study villages were larger than other communities in the HDSS and only households with two or more children were included in the trial. ${ }^{4}$ Thus, the results from this study may not be generalisable to children from smaller households or smaller communities. These findings also may not be generalisable outside of regions with similar seasonal variation in food availability. This study was conducted over a span of only 35 days. Although the focus was to evaluate short-term weight gain in children, a longer time period may reflect more accurate weight change, and longer-term data would be useful to understand modifiable risk factors for nutritional outcomes. Future studies could evaluate weight changes over an entire lean season to understand the total effect of the lean season on nutrition outcomes. Children included in this analysis were participating in a trial of antibiotics on the intestinal microbiome. Antibiotics may disrupt the paediatric microbiome and affect weight gain outcomes. ${ }^{15} 19$ However, all predictors were measured at baseline prior to randomisation, and we do not anticipate that they were different across randomisation arms, and treatment arm was included as a covariate in models. Few children were given antibiotics outside of the study treatment during the course of the study. Such antibiotic use may be influenced by baseline characteristics and could potentially be a mediator of any effect of baseline characteristics on nutritional outcomes. Given that antibiotic use in this study area was higher than would be anticipated outside of a trial of antibiotics, ${ }^{20}$ generalisability may be limited in settings where antibiotic use is very low. Finally, the sample size of this study was limited. Larger prospective studies would have greater power to identify potential risk factors for low weight gain.
In this study, we demonstrated that experiences of household food insecurity prior to the lean season are associated with decreased weight gain in children in rural Burkina Faso during the lean season. Children are particularly vulnerable to adverse nutrition outcomes during this period, and this study suggests that interventions that address food insecurity may be effective for reducing the incidence of malnutrition during the lean season. Given that the determinants of weight gain may differ in different seasons, such policies should consider the seasonal variation of crops in agrarian communities and target interventions during the months prior to the vulnerable season when malnutrition may develop.

\section{Author affiliations}

${ }^{1}$ Francis I Proctor Foundation, University of California, San Francisco, California, USA ${ }^{2}$ Centre de Recherche en Sante de Nouna, Nouna, Burkina Faso

${ }^{3}$ Institute of Public Health, Heidelberg University, Heidelberg, Germany

${ }^{4}$ Africa Health Research Institute, Somkhele, South Africa

${ }^{5}$ Department of Global Health and Population, Harvard T.H. Chan School of Public Health, Boston, Massachusetts, United States

${ }^{6}$ Department of Ophthalmology, University of California, San Francisco, California, United States

${ }^{7}$ Department of Epidemiology and Biostatistics, University of California, San Francisco, California, United States

Contributors EGD: formulate research question, data analysis, writing the article. AS: formulating research question, designing study, implementation of study, critical review of article. LO: designing study, implementation of study, critical review of article. CD: designing study, implementation of study, critical review of article. CT: designing study, implementation of study, critical review of article. PZ: designing study, implementation of study, data analysis, critical review of article. TB: formulating research question, designing study, critical review of article. KSO: data analysis, critical review of article. EL: designing study, implementation of study, critical review of article. JDK: formulate research question, designing study, implementation of study, critical review of article. CEO: formulate research question, designing study, implementation of study, data analysis, writing article.

Funding This work was supported in part by a Research to Prevent Blindness Career Development Award to CEO.

Competing interests None declared.

Patient consent for publication Not required.

Ethics approval This study was reviewed and approved by the Committee on Human Research at the University of California, San Francisco (Protocol 17-22036) and the Comité Institutionnel d'Ethique at the Centre de Recherche en Santé de Nouna (CRSN; Protocol 2017-05-/CIE/CRSN).

Provenance and peer review Not commissioned; externally peer reviewed.

Data availability statement No data are available.

Open access This is an open access article distributed in accordance with the Creative Commons Attribution Non Commercial (CC BY-NC 4.0) license, which permits others to distribute, remix, adapt, build upon this work non-commercially, and license their derivative works on different terms, provided the original work is properly cited, appropriate credit is given, any changes made indicated, and the use is non-commercial. See: http://creativecommons.org/licenses/by-nc/4.0/.

\section{REFERENCES}

1. Rice AL, Sacco L, Hyder A, et al. Malnutrition as an underlying cause of childhood deaths associated with infectious diseases in developing countries. Bull World Health Organ 2000;78:1207-21.

2. Black RE, Victora CG, Walker SP, et al. Maternal and child undernutrition and overweight in low-income and middle-income countries. Lancet 2013;382:427-51.

3. Arimond M, Ruel MT. Dietary diversity is associated with child nutritional status: evidence from 11 demographic and health surveys. J Nutr 2004;134:2579-85. 
4. Sie A, Tapsoba C, Dah C, et al. Dietary diversity and nutritional status among children in rural Burkina Faso. Int Health 2018;382:426-7.

5. Rogawski McQuade ET, Clark S, Bayo E, et al. Seasonal food insecurity in Haydom, Tanzania, is associated with low birthweight and acute malnutrition: results from the MAL-ED study. Am J Trop Med Hyg 2019;100:681-7.

6. Hillbruner C, Egan R. Seasonality, household food security, and nutritional status in Dinajpur, Bangladesh. Food Nutr Bull 2008;29:221-31.

7. Arsenault JE, Nikiema L, Allemand P, et al. Seasonal differences in food and nutrient intakes among young children and their mothers in rural Burkina Faso. J Nutr Sci 2014;3.

8. Somé JW JA. The influence of crop production and socioeconomic factors on seasonal household dietary diversity in Burkina Faso. PLoS One J 2018.

9. Sié A, Louis V, Gbangou A, et al. The health and demographic surveillance system (HDSS) in Nouna, Burkina Faso, 1993-2007. Glob Health Action 2010;3:5284.

10. Oldenburg CE, Sié A, Coulibaly B, et al. Effect of commonly used pediatric antibiotics on gut microbial diversity in preschool children in Burkina Faso: a randomized clinical trial. Open Forum Infect Dis. In Press 2018;5.

11. Sié A, Dah C, Ouermi L, et al. Effect of antibiotics on short-term growth among children in Burkina Faso: a randomized trial. Am J Trop Med Hyg 2018;99:789-96.

12. WHO Multicentre Growth Reference Study Group. WHO child growth standards: Length/height-for-age, weight-for-age, weight-for-length, weight-for-height and body mass index-for-age: methods and development. 312. Geneva World Heal Organ, 2006.

13. Kennedy G, Ballard T, Dop M. Guidelines for measuring household and individual dietary diversity. food and agriculture organization of the United nations, 2011. Available: http://www.fao.org/3/a-i1983e.pdf

14. Knueppel D, Demment M, Kaiser L. Validation of the household food insecurity access scale in rural Tanzania 2009;13:360-7.

15. Doan T, Arzika AM, Ray KJ, et al. Gut microbial diversity in AntibioticNaive children after systemic antibiotic exposure: a randomized controlled trial. Clin Infect Dis 2017;64:1147-53.

16. Santos LP, Gigante DP. Relationship between food insecurity and nutritional status of Brazilian children under the age of five. Rev Bras Epidemiol 2013;16:984-94.

17. Becquey E, Delpeuch F, Konaté AM, et al. Seasonality of the dietary dimension of household food security in urban Burkina Faso. $\mathrm{Br} J$ Nutr 2012;107:1860-70.

18. Akombi BJ, Agho KE, Merom D, et al. Child malnutrition in subSaharan Africa: a meta-analysis of demographic and health surveys (2006-2016). PLoS One 2017;12:e0177338.

19. Bokulich NA, Chung J, Battaglia T, et al. Antibiotics, birth mode, and diet shape microbiome maturation during early life. Sci Trans/ Med 2016;8:343ra82.

20. Sie A, Coulibaly B, Adama S, et al. Antibiotic prescriptions among children younger than 5 years in Nouna district, Burkina Faso. Am J Trop Med Hyg. In Press 2019. 